\title{
Distribuição bivariada gama beta II: soma, produto e proporção das variáveis componentes
}

A.P.M. SILVA ${ }^{1}$ UFSJ - Universidade Federal de São João del-Rei, Campus de Sete Lagoas, CSL, Cx. P. 56, 35701-970 Sete Lagoas, MG, Brasil

J.A. RODRIGUES'2, DEPEN - Departamento de Ensino, IFBA - Instituto Federal de Educação, Ciência e Tecnologia da Bahia, 45823-431 Eunápolis, BA, Brasil

L.M. CHAVES 3 , DEX - Departamento de Ciências Exatas, UFLA - Universidade Federal de Lavras, Cx.P. 3037, 37200-000 Lavras, MG, Brasil

D.J. SOUZA 4 , DEX - Departamento de Ciências Exatas, UFLA - Universidade Federal de Lavras, Cx.P. 3037, 37200-000 Lavras, MG, Brasil.

Resumo. Os modelos bivariados tem sido utilizados com sucesso na análise de processos hidrológicos. Neste trabalho, são deduzidas as distribuições exatas das variáveis $U=X+Y, P=X Y$ e $Q=X /(X+Y)$ juntamente com seus respectivos momentos quando $X$ e $Y$ seguem o modelo bivariado gama beta II. Essas funções descrevem importantes variáveis hidrológicas. Os resultados obtidos são aplicados em dados de precipitações pluviométricas ocorridas na cidade de Passo Fundo - RS.

Palavras-chave. Distribuição gama beta II, combinação de variáveis aleatórias, precipitação pluviométrica.

\section{Introdução}

Motivados pelo crescente uso principalmente na análise de dados não normais, vários modelos bivariados podem ser encontrados na literatura [2]. Os trabalhos desenvolvidos por [1] impulsionaram o uso da distribuição gama bivariada na análise de processos hidrológicos, sendo atualmente um dos mais utilizados. Por exemplo, [15] estuda a aplicabilidade da distribuição gama bivariada de Smith na análise de frequência das variáveis hidrológicas duração e volume; [16] apresentam uma revisão de vários modelos gama, com diferentes parâmetros de escala e forma, apontando as vantagens e desvantagens de cada modelo no estudo de precipitação; [9] estudam o comportamento de dados de seca do Estado de Nebraska considerando a

\footnotetext{
${ }^{1}$ anapaula@ufsj.edu.br

2 jailsondearaujo@yahoo.com.br

3 lucas@dex.ufla.br

${ }^{4}$ devaniljaques@dex.ufla.br
} 
distribuição gama bivariada de Cherian e [10] realizam o mesmo estudo com a distribuição exponencial bivariada de Friday e Patil; [7] aplica um modelo bivariado gama exponencial na modelagem de dados de seca.

Considerando que $X$ e $Y$ se distribuem segundo um modelo bivariado, funções dessas variáveis aleatórias, expressas por $U=X+Y, P=X Y$ e $Q=X /(X+Y)$, têm um significado físico importante de modo que diferentes autores têm trabalhado no sentido de caracterizar essas distribuições e aplicá-las em diferentes áreas do conhecimento, em particular em hidrologia $[6,11,4,8,14]$. Por exemplo, se $X$ representa o período de chuva e $Y$ o período contíguo sem ocorrência de chuva, $U=X+Y$ denota o período climático e $Q=X /(X+Y)$ a proporção de chuva. Neste contexto, o trabalho têm por objetivo apresentar a distribuição bivariada Gama Beta tipo II e deduzir a distribuição exata das variáveis $U=X+Y, P=$ $X Y$ e $Q=X /(X+Y)$ sob a pressuposição de que $X$ e $Y$ seguem esse modelo. Como aplicação é realizado o ajuste dessas distribuições a dados de precipitação pluviométrica do município de Passo Fundo, RS.

\subsection{Distribuições beta}

Distribuição beta tipo I: Uma variável aleatória $X$ tem distribuição beta tipo I com parâmetros $\alpha>0$ e $\beta>0$ quando sua função densidade de probabilidade (fdp) é da forma:

$$
f(x)=\frac{x^{\alpha-1}(1-x)^{\beta-1}}{B(\alpha, \beta)},
$$

em que $0<x<1$ e $B(\alpha, \beta)$ representa a função beta,

$$
B(\alpha, \beta)=\int_{0}^{1} t^{\alpha-1}(1-t)^{\beta-1} d t .
$$

Simbolicamente, quando $X$ possui função densidade de probabilidade dada por (1.1), denotamos por $X \sim B I(\alpha, \beta)$, ou apenas, $X \sim B(\alpha, \beta)$.

Distribuição beta tipo II: Uma variável aleatória $X$ tem distribuição beta tipo II com parâmetros $\alpha>0, \beta>0$ e $\lambda>0$ quando sua função densidade de probabilidade (fdp) é da forma:

$$
f(x)=\frac{\lambda^{\beta}}{\mathrm{B}(\alpha, \beta)} x^{\alpha-1}(\lambda+x)^{-(\alpha+\beta)},
$$

em que $x>0$. Quando $X$ possui função densidade de probabilidade dada por (1.3), denotamos por $X \sim B I I(\alpha, \beta)$.

As duas distribuições beta estão relacionadas intrinsecamente por meio de uma transformação. Várias características referentes aos dois modelos podem ser encontradas em $[3,5]$.

Além da função beta, os cálculos envolvidos no trabalho incluem o uso de outras funções especiais como a função gama,

$$
\Gamma(\alpha)=\int_{0}^{\infty} t^{\alpha-1} \exp (-t) d t
$$


a função $\psi(\cdot)$,

$$
\psi(\alpha)=\frac{d}{d \alpha}[\ln \Gamma(\alpha)],
$$

a função hipergeométrica confluente,

$$
{ }_{1} F_{1}(a ; b ; x)=\frac{\Gamma(b)}{\Gamma(b-a) \Gamma(a)} \int_{0}^{1} t^{a-1}(1-t)^{b-a-1} \exp (-x t) d t,
$$

com $0<a<b$ e $b \neq 0,-1,-2, \ldots$ e a função cilíndrica parabólica,

$$
D_{v}(x)=\frac{2^{v / 2} \exp \left(-x^{2} / 4\right)}{\Gamma(-v / 2)} \int_{0}^{\infty} t^{-(1+v / 2)}(1+t)^{v / 2-1 / 2} \exp \left(\frac{-x^{2} t}{2}\right) d t .
$$

As propriedades dessas funções especiais podem ser vistas em $[3,12]$. Serão ainda utilizados os importantes Lemas 1.1 e 1.2.

Lema 1.1. (Equação 2.3.6.1, [13]). Se $a>0$,

$$
\int_{0}^{a} x^{\alpha-1}(a-x)^{\beta-1} \exp (-p x) d x=\mathrm{B}(\alpha, \beta) a^{\alpha+\beta-1}{ }_{1} F_{1}(\alpha ; \alpha+\beta ;-a p) .
$$

Lema 1.2. (Equação 2.3.15.1, [13]). Se $\alpha>0$ e $\beta>0$,

$$
\int_{0}^{\infty} x^{\alpha-1} \exp \left(-r x^{2}-q x\right) d x=\Gamma(\alpha)(2 r)^{-\alpha / 2} \exp \left(\frac{q^{2}}{8 r}\right) D_{-\alpha}\left(\frac{q}{\sqrt{2 r}}\right) .
$$

\section{O modelo}

A distribuição bivariada gama beta tipo II tem função densidade de probabilidade (fdp) conjunta dada por:

$$
f_{X, Y}(x, y)=K x^{\alpha-1} y^{\beta-1} \exp [-(a x+c x y)]
$$

com $x>0, y>0, a>0, c>0,0<\beta<\alpha$ e $K$ é a constante de normalização definida por

$$
K=\frac{c^{\beta} a^{\alpha-\beta}}{\Gamma(\beta) \Gamma(\alpha-\beta)} .
$$

Esse modelo pertence à família gama bivariada de Arnould (ver [2]) e com essa parametrização ainda não foi abordado na literatura. 


\subsection{Funções Densidade de Probabilidade}

Considerando que $X$ e $Y$ têm distribuição conjunta dada pelo modelo (2.1), as fdps marginais de $X$ e $Y$ são respectivamente:

$$
\begin{aligned}
f_{X}(x) & =\int_{0}^{\infty} f(x, y) d y=\int_{0}^{\infty} K x^{\alpha-1} y^{\beta-1} \exp [-(a x+c x y)] d y \\
& =\frac{a^{\alpha-\beta}}{\Gamma(\alpha-\beta)} x^{\alpha-\beta-1} \exp (-a x), \\
f_{Y}(y) & =\int_{0}^{\infty} f(x, y) d x=\int_{0}^{\infty} K x^{\alpha-1} y^{\beta-1} \exp [-(a x+c x y)] d x \\
& =\frac{c^{\beta} a^{\alpha-\beta} \Gamma(\alpha)}{\Gamma(\beta) \Gamma(\alpha-\beta)} y^{\beta-1}\left[c\left(\frac{a}{c}+y\right)\right]^{-\alpha} \\
& =\frac{(a / c)^{\alpha-\beta}}{\mathrm{B}(\beta, \alpha-\beta)} y^{\beta-1}\left(\frac{a}{c}+y\right)^{-\alpha} .
\end{aligned}
$$

Portanto, $X \sim G(\alpha-\beta, a)$ e $Y \sim \operatorname{BII}(\beta, \alpha-\beta, a / c)$.

Nos Teoremas 2.1, 2.2 e 2.3 são deduzidas as fdps das variáveis $U=X+Y$, $P=X Y$ e $Q=X /(X+Y)$ quando $X$ e $Y$ seguem o modelo (2.1). Inicialmente apresentamos a fdp conjunta das variáveis $U$ e $Q$ (ver equação 2.5) e a fdp conjunta das variáveis $X$ e $P$ (ver equação 2.7), que são úteis nas demonstrações desses teoremas.

Considere $(U, Q)=(X+Y, X /(X+Y))$, onde $X$ e $Y$ são variáveis aleatórias com função densidade de probabilidade conjunta dada pela equação (2.1). Então, o Jacobiano da transformação é dado por:

$$
J=\left|\begin{array}{ll}
\frac{\partial u}{\partial x} & \frac{\partial u}{\partial y} \\
\frac{\partial q}{\partial x} & \frac{\partial q}{\partial y}
\end{array}\right|=\left|\begin{array}{cc}
1 & 1 \\
\frac{y}{(x+y)^{2}} & -\frac{x}{(x+y)^{2}}
\end{array}\right|=-\frac{1}{x+y} .
$$

Assim, a fdp conjunta de $(U, Q)=(X+Y, X /(X+Y))$ é dada por

$$
\begin{aligned}
f_{U, Q}(u, q) & =f_{X, Y}(u q, u(1-q)) \times|J|^{-1} \\
& =K u^{\alpha+\beta-1} q^{\alpha-1}(1-q)^{\beta-1} \exp \left\{-a u q-c u^{2} q(1-q)\right\} \\
& =K u^{\alpha+\beta-1} q^{\alpha-1}(1-q)^{\beta-1} \exp \left\{-(a+c u) u q+c u^{2} q^{2}\right\} \\
& =K u^{\alpha+\beta-1} q^{\alpha-1}(1-q)^{\beta-1} \exp \left\{c u^{2}(q-\theta)^{2}-\frac{(a+c u)^{2}}{4 c}\right\}
\end{aligned}
$$


Considere agora $(X, P)=(X, X Y)$, onde $X$ e $Y$ são variáveis aleatórias com função densidade de probabilidade conjunta dada pela equação (2.1). Então, o Jacobiano da transformação é dado por:

$$
J=\left|\begin{array}{ll}
\frac{\partial x}{\partial x} & \frac{\partial x}{\partial y} \\
\frac{\partial p}{\partial x} & \frac{\partial p}{\partial y}
\end{array}\right|=\left|\begin{array}{cc}
1 & 0 \\
y & x
\end{array}\right|=x .
$$

Assim, a fdp conjunta de $(X, P)$ é dada por

$$
\begin{aligned}
f_{X, P}(x, p) & =f_{X, Y}\left(x, \frac{p}{x}\right) \times|J|^{-1} \\
& =K x^{\alpha-\beta-1} p^{\beta-1} \exp [-(a x+c p)] .
\end{aligned}
$$

Teorema 2.1. Sejam $X$ e $Y$ variáveis aleatórias com função densidade de probabilidade conjunta dada pela equação (2.1) e U variável aleatória dada por $U=X+Y$. Se $\alpha \geq 2$ e $\beta \geq 2$ são inteiros, então:

$$
\begin{aligned}
f_{U}(u)= & K u^{\alpha+\beta-1} \exp \left\{-\frac{(a+c u)^{2}}{4 c}\right\} \sum_{m=0}^{\alpha-1} \sum_{n=0}^{\beta-1} \sum_{j=0}^{n}\left(\begin{array}{c}
n \\
j
\end{array}\right)\left(\begin{array}{c}
\beta-1 \\
n
\end{array}\right)\left(\begin{array}{c}
\alpha-1 \\
m
\end{array}\right) \times \\
& \frac{\theta^{\alpha+n-(m+j+1)}}{m+j+1}\left[\theta^{m+j-1}{ }_{1} F_{1}\left(\frac{m+j+1}{2} ; \frac{m+j+3}{2} ; c u^{2} \theta^{2}\right)+\right. \\
& \left.(1-\theta)^{m+j-1}{ }_{1} F_{1}\left(\frac{m+j+1}{2} ; \frac{m+j+3}{2} ; c u^{2}(1-\theta)^{2}\right)\right],
\end{aligned}
$$

para $0 \leq \theta \leq 1 e$

$$
\begin{aligned}
f_{U}(u)= & K u^{\alpha+\beta-1} \exp \left\{-\frac{(a+c u)^{2}}{4 c}\right\} \sum_{m=0}^{\alpha-1} \sum_{n=0}^{\beta-1} \sum_{j=0}^{n}\left(\begin{array}{c}
n \\
j
\end{array}\right)\left(\begin{array}{c}
\beta-1 \\
n
\end{array}\right)\left(\begin{array}{c}
\alpha-1 \\
m
\end{array}\right) \times \\
& \frac{\theta^{\alpha+n-(m+j+1)}}{m+j+1}\left[(1-\theta)^{m+j-1}{ }_{1} F_{1}\left(\frac{m+j+1}{2} ; \frac{m+j+3}{2} ; c u^{2}(1-\theta)^{2}\right)-\right. \\
& \left.\theta^{m+j-1}{ }_{1} F_{1}\left(\frac{m+j+1}{2} ; \frac{m+j+3}{2} ; c u^{2} \theta^{2}\right)\right],
\end{aligned}
$$

para $\theta>1$, em que $u>0$ e $\theta=(a-c u) /(2 c u)$.

Demonstração. Considere $U$ e $P$ variáveis aleatórias com função densidade de probabilidade conjunta dada pela equação (2.5). Para $0 \leq \theta \leq 1$ então a fdp de $U$ é dada por:

$$
\begin{aligned}
f_{U}(u) & =K u^{\alpha+\beta-1} \int_{0}^{1} q^{\alpha-1}(1-q)^{\beta-1} \exp \left\{c u^{2}(q-\theta)^{2}-\frac{(a+c u)^{2}}{4 c}\right\} d q \\
& =K u^{\alpha+\beta-1} \exp \left\{-\frac{(a+c u)^{2}}{4 c}\right\} \int_{0}^{1} q^{\alpha-1}(1-q)^{\beta-1} \exp \left\{c u^{2}(q-\theta)^{2}\right\} d q .
\end{aligned}
$$


Fazendo $y=(w-\theta)^{2}$ temos:

$$
\begin{aligned}
& f_{U}(u)=2^{-1} K u^{\alpha+\beta-1} \exp \left\{-\frac{(a+c u)^{2}}{4 c}\right\}\left(\int_{0}^{\theta^{2}}+\int_{0}^{(1-\theta)^{2}}\right)(\sqrt{y}+\theta)^{\alpha-1} \times \\
& (1-\sqrt{y}-\theta)^{\beta-1} \exp \left\{c u^{2} y\right\} y^{-1 / 2} d y \\
& =2^{-1} K u^{\alpha+\beta-1} \exp \left\{-\frac{(a+c u)^{2}}{4 c}\right\}\left(\int_{0}^{\theta^{2}}+\int_{0}^{(1-\theta)^{2}}\right) \sum_{m=0}^{\alpha-1}\left(\begin{array}{c}
\alpha-1 \\
m
\end{array}\right)(\sqrt{y})^{m} \theta^{\alpha-m-1} \times \\
& \sum_{n=0}^{\beta-1}\left(\begin{array}{c}
\beta-1 \\
n
\end{array}\right)(\sqrt{y}+\theta)^{n} \exp \left\{c u^{2} y\right\} y^{-1 / 2} d y \\
& =2^{-1} K u^{\alpha+\beta-1} \exp \left\{-\frac{(a+c u)^{2}}{4 c}\right\} \sum_{m=0}^{\alpha-1} \sum_{n=0}^{\beta-1} \sum_{j=0}^{n}\left(\begin{array}{c}
n \\
j
\end{array}\right)\left(\begin{array}{c}
\beta-1 \\
n
\end{array}\right)\left(\begin{array}{c}
\alpha-1 \\
m
\end{array}\right) \times \\
& \frac{\theta^{\alpha+n-(m+j+1)}}{m+j+1}\left(\int_{0}^{\theta^{2}}+\int_{0}^{(1-\theta)^{2}}\right) y^{\frac{m+j-1}{2}} \exp \left\{c u^{2} y\right\} d y \text {. }
\end{aligned}
$$

Aplicando o Lema (1.1) à integral acima, obtém-se:

$$
\begin{aligned}
f_{U}(u)=K u^{\alpha+\beta-1} \exp \left\{-\frac{(a+c u)^{2}}{4 c}\right\} \sum_{m=0}^{\alpha-1} \sum_{n=0}^{\beta-1} \sum_{j=0}^{n}\left(\begin{array}{c}
n \\
j
\end{array}\right)\left(\begin{array}{c}
\beta-1 \\
n
\end{array}\right)\left(\begin{array}{c}
\alpha-1 \\
m
\end{array}\right) \times \\
\frac{\theta^{\alpha+n-(m+j+1)}}{m+j+1}\left[\theta^{m+j-1}{ }_{1} F_{1}\left(\frac{m+j+1}{2} ; \frac{m+j+3}{2} ; c u^{2} \theta^{m+j-1}\right)+\right. \\
\left.(1-\theta)^{m+j-1}{ }_{1} F_{1}\left(\frac{m+j+1}{2} ; \frac{m+j+3}{2} ; c u^{2}(1-\theta)^{m+j-1}\right)\right] .
\end{aligned}
$$

O resultado para $\theta>1$ é obtido similarmente.

Teorema 2.2. Sejam $X$ e $Y$ variáveis aleatórias com função densidade de probabilidade conjunta dada pela equação (2.1) e $Q$ a variável aleatória dada por $Q=X /(X+Y)$. Se $\alpha \geq 2$ e $\beta \geq 2$ são inteiros, então:

$$
\begin{aligned}
f_{Q}(q)= & K q^{\alpha-1}(1-q)^{\beta-1} \Gamma(\alpha+\beta)[2 c q(1-q)]^{-\frac{\alpha+\beta}{2}} \exp \left[\frac{(a q)^{2}}{8 c q(1-q)}\right] \times \\
& D_{-(\alpha+\beta)}\left(\frac{a q}{\sqrt{2 c q(1-q)}}\right),
\end{aligned}
$$

em que $0<q<1$.

Demonstração. De (2.5) tem-se que a distribuição de $Q$ é dada por:

$$
f_{Q}(q)=\int_{0}^{\infty} f(u, q) d u
$$

TEMA Tend. Mat. Apl. Comput., 14, No. 1 (2013), 95-108. doi: 10.5540/tema.2013.014.01.0095 


$$
=K q^{\alpha-1}(1-q)^{\beta-1} \int_{0}^{\infty} u^{\alpha+\beta-1} \exp \left\{-\left[a u q+c q(1-q) u^{2}\right]\right\} d u .
$$

O resultado segue da aplicação do Lema (1.2) à integral.

Teorema 2.3. Sejam $X$ e $Y$ variáveis aleatórias com função densidade de probabilidade conjunta dada pela equação (2.1) e $P$ a variável aleatória dada por $P=X Y$. Se $\alpha \geq 2$ e $\beta \geq 2$ são inteiros, então:

$$
f_{P}(p)=\frac{c^{\beta}}{\Gamma(\beta)} p^{\beta-1} \exp (-c p),
$$

em que $p>0$.

Demonstração. Considere $X$ e $P$ variáveis aleatórias com função densidade de probabilidade conjunta dada pela equação (2.7). A fdp de $P$ é dada por

$$
\begin{aligned}
f_{P}(p) & =\int_{0}^{\infty} f(x, p) d x \\
& =\int_{0}^{\infty} K x^{\alpha-\beta-1} p^{\beta-1} \exp [-(a x+c p)] d x \\
& =K p^{\beta-1} \exp (-c p) \int_{0}^{\infty} x^{\alpha-\beta-1} \exp (-a x) d x \\
& =\frac{c^{\beta}}{\Gamma(\beta)} p^{\beta-1} \exp (-c p) .
\end{aligned}
$$

\subsection{Momentos}

Nesta seção são deduzidos os momentos das variáveis $U=X+Y$ e $P=X Y$ quando $X$ e $Y$ seguem o modelo gama beta II (ver equação 2.1). Inicialmente apresentamos o Lema 2.1 o qual é muito útil nas demonstrações dos Teoremas 2.4 e 2.5.

Lema 2.1. Sejam $X$ e $Y$ variáveis aleatórias com função densidade de probabilidade conjunta dada pela equação (2.1). Então:

$$
E\left[X^{n} Y^{m}\right]=\frac{a^{m-n} \Gamma(\beta+m) \Gamma(\alpha+n-\beta-m)}{c^{m} \Gamma(\beta) \Gamma(\alpha-\beta)},
$$

para os inteiros $n \geq 1$ e $1 \leq m \leq n$. 
Demonstração. Sob o modelo (2.1), tem-se que:

$$
\begin{aligned}
E\left[X^{n} Y^{m}\right] & =\int_{0}^{\infty} \int_{0}^{\infty} x^{n} y^{m} f(x, y) d y d x \\
& =\int_{0}^{\infty} \int_{0}^{\infty} K x^{\alpha+n-1} y^{\beta+m-1} \exp [-(a x+c x y)] d y d x \\
& =K \int_{0}^{\infty} x^{\alpha+n-1} \exp (-a x)\left[\int_{0}^{\infty} y^{\beta+m-1} \exp (-c x y) d y\right] d x \\
& =K \int_{0}^{\infty} x^{\alpha+n-1} \exp (-a x) \frac{\Gamma(\beta+m)}{(c x)^{\beta+m}} d x \\
& =\frac{a^{m-n} \Gamma(\beta+m) \Gamma(\alpha+n-\beta-m)}{c^{m} \Gamma(\beta) \Gamma(\alpha-\beta)} \quad \text { com } m \leq n .
\end{aligned}
$$

Em particular, para $n=m=1$ obtém-se:

$$
E[X Y]=\frac{\Gamma(\beta+1) \Gamma(\alpha-\beta)}{c \Gamma(\beta) \Gamma(\alpha-\beta)}=\frac{\beta}{c} .
$$

Teorema 2.4. Sejam $X$ e $Y$ variáveis aleatórias com função densidade de probabilidade conjunta dada pela equação (2.1). Então:

$$
E\left[U^{n}\right]=\sum_{j=0}^{n}\left(\begin{array}{c}
n \\
j
\end{array}\right) \frac{a^{n} \Gamma(\beta+n-j) \Gamma(\alpha+2 j-\beta)}{c^{n-j} \Gamma(\beta) \Gamma(\alpha-\beta)},
$$

para todo inteiro $n \geq 1$.

Demonstração. Temos que:

$$
E\left[U^{n}\right]=E\left[(X+Y)^{n}\right]=\sum_{j=0}^{n}\left(\begin{array}{c}
n \\
j
\end{array}\right) E\left[X^{j} Y^{n-j}\right] .
$$

O resultado segue da aplicação do Lema (2.1).

Teorema 2.5. Sejam $X$ e $Y$ variáveis aleatórias com função densidade de probabilidade conjunta dada pela equação (2.1). Então:

$$
E\left[P^{n}\right]=\frac{\Gamma(\beta+n)}{c^{n} \Gamma(\beta)}
$$

para todo inteiro $n \geq 1$.

Demonstração. Temos que: $E\left[P^{n}\right]=E\left[(X Y)^{n}\right]=E\left[X^{n} Y^{n}\right]$. O resultado segue da aplicação do Lema (2.1) para $m=n$. 


\subsection{Estimação}

Seja $\left(x_{1}, y_{1}\right),\left(x_{2}, y_{2}\right), \ldots,\left(x_{n}, y_{n}\right)$ uma amostra aleatória da variável aleatória $(X, Y)$ com distribuição conjunta expressa por (2.1), de parâmetros $a, c, \alpha$ e $\beta$. A função de verossimilhança é:

$$
\begin{aligned}
L & =\prod_{i=1}^{n} f\left(x_{i}, y_{i}\right)=\prod_{i=1}^{n} K x_{i}^{\alpha-1} y_{i}^{\beta-1} \exp \left[-\left(a x_{i}+c x_{i} y_{i}\right)\right] \\
& =K^{n} \prod_{i=1}^{n}\left(x_{i}^{\alpha-1} y_{i}^{\beta-1}\right) \exp \left[-\left(a \sum_{i=1}^{n} x_{i}+c \sum_{i=1}^{n} x_{i} y_{i}\right)\right] .
\end{aligned}
$$

Aplicando a função logarítmica a ambos os lados da equação (2.9) obtém-se:

$$
\ln (L)=n \ln (K)+(\alpha-1) \sum_{i=1}^{n} \ln \left(x_{i}\right)+(\beta-1) \sum_{i=1}^{n} \ln \left(y_{i}\right)-a \sum_{i=1}^{n} x_{i}-c \sum_{i=1}^{n} x_{i} y_{i} .
$$

Assim,

$$
\begin{aligned}
& \frac{\partial \ln (L)}{\partial a}=\frac{n(\alpha-\beta)}{a}-\sum_{i=1}^{n} x_{i}, \\
& \frac{\partial \ln (L)}{\partial c}=\frac{n \beta}{c}-\sum_{i=1}^{n} x_{i} y_{i}, \\
& \frac{\partial \ln (L)}{\partial \alpha}=n \ln (a)-n \psi(\alpha-\beta)+\sum_{i=1}^{n} \ln \left(x_{i}\right), \\
& \frac{\partial \ln (L)}{\partial \beta}=n \ln (c)-n \ln (a)-n \psi(\beta)+n \psi(\alpha-\beta)+\sum_{i=1}^{n} \ln \left(y_{i}\right),
\end{aligned}
$$

em que $\psi(\cdot)$ denota a função psi dada pela equação (1.5).

Os estimadores de máxima verossimilhança dos parâmetros $a, c, \alpha$ e $\beta$, são os valores na qual a função de verossimilhança é máxima, ou seja, é a solução do sistema de equações:

$$
\left\{\begin{array}{l}
\frac{n(\hat{\alpha}-\hat{\beta})}{\hat{a}}-\sum_{i=1}^{n} x_{i}=0 \\
\frac{n \hat{\beta}}{\hat{c}}-\sum_{i=1}^{n} x_{i} y_{i}=0 \\
n \ln (\hat{a})-n \psi(\hat{\alpha}-\hat{\beta})+\sum_{i=1}^{n} \ln \left(x_{i}\right)=0 \\
n \ln (\hat{c})-n \ln (\hat{a})-n \psi(\hat{\beta})+n \psi(\hat{\alpha}-\hat{\beta})+\sum_{i=1}^{n} \ln \left(y_{i}\right)=0 .
\end{array}\right.
$$

A solução do sistema de equações de máxima verossimilhança pode ser obtida numericamente através do software de computação algébrica MAPLE (versão 16.02). 
Como não é possível obter as propriedades exatas do estimador de máxima verossimilhança, um estudo computacional é apresentado. Para os dados utilizados na seção 3 seguinte, as estimativas de máxima verossimilhança para os parâmetros do modelo são:

$$
(\widehat{a}, \widehat{c}, \widehat{\alpha}, \widehat{\beta})=(0.8654,0.1899,3.3920,1.2842)
$$

Fixando-se três dessas estimativas, é possível simular o gráfico do erro quadrático médio (EQM) do estimador de máxima verossimilhança da seguinte forma: Suponha fixados os valores de $\widehat{c}=0.1899, \widehat{\alpha}=3.3920$ e $\widehat{\beta}=1.2842$, e permita que $a$ varie no intervalo $[0,64905,1.752436]$. Tomam-se valores $a_{i}(i=1,2, \ldots I)$ nesse intervalo e, para cada um desses valores, considera-se que o vetor dos parâmetros reais é $\left(a_{i}, \widehat{c}, \widehat{\alpha}, \widehat{\beta}\right)=\left(a_{i}, 0.1899,3.3920,1.2842\right)$. Substituindo na densidade (2.1), uma amostra de tamanho 101 é obtida. A partir desta, a solução do sistema das equações de máxima verossimilhança é obtido e o erro quadrático determinado. Esse procedimento, repetido 1000 vezes, permite a obtenção de uma estimativa para o erro quadrático médio. Com isto, é possível traçar o gráfico do erro quadrático médio como função do parâmetro $a$. Repetindo-se o processo para os outros parâmetros, obtém-se gráficos representados pelas Figuras 1, 2, 3 e 4.

Supondo os parâmetros reais dados por $(a, c, \alpha, \beta)=(0.8654,0.1899,3.3920,1.2842)$ foram simuladas 100.000 amostras de tamanho $101 \mathrm{e}$, para cada amostra, calculadas as estimativas de máxima verossimilhança dos parâmetros, $(\widehat{a}, \widehat{c}, \widehat{\alpha}, \widehat{\beta})$. Com isso foi possível obter:

1. As esperanças de cada componente dos estimadores:

$$
(E[\widehat{a}], E[\widehat{c}], E[\widehat{\alpha}], E[\widehat{\beta}])=(1.52940,0.45604,4.23305,0.74029)
$$

2. O erro quadrático médio:

$$
\begin{aligned}
E Q M & =\frac{1}{100000} \sum\left[(a-\widehat{a})^{2}+(b-\widehat{b})^{2}+(\alpha-\widehat{\alpha})^{2}+(\beta-\widehat{\beta})^{2}\right] \\
& =0.49260+0.07933+1.02017+0.30527=1.89736
\end{aligned}
$$

3. A Variância das estimativas:

$$
\begin{aligned}
\operatorname{Var} & =\frac{1}{100000} \sum\left[(\widehat{a}-E[\widehat{a}])^{2}+(\widehat{b}-E[\widehat{b}])^{2}+(\widehat{\alpha}-[\widehat{\alpha}])^{2}+(\widehat{\beta}-E[\widehat{\beta}])^{2}\right] \\
& =0.05170+0.07933+1.02017+0.03053=0.38242
\end{aligned}
$$

4. O Viés:

$$
\begin{aligned}
\text { Vies } & =(a-E[\widehat{a}])^{2}+(b-E[\widehat{b}])^{2}+(\alpha-E[\widehat{\alpha}])^{2}+(\beta-E[\widehat{\beta}])^{2} \\
& =0.44089+0.07083+0.70741+0.29581=1.51494
\end{aligned}
$$

Observe que, como tem que ser, $E Q M=$ Var + Vies. 


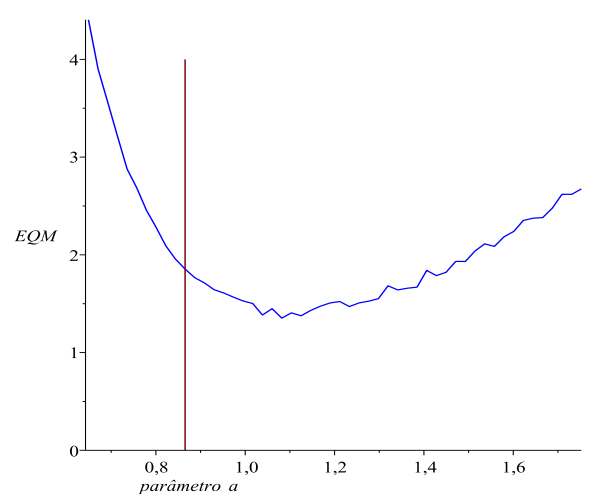

Figura 1: $(a, 0.1899,3.3920,1.2842)$

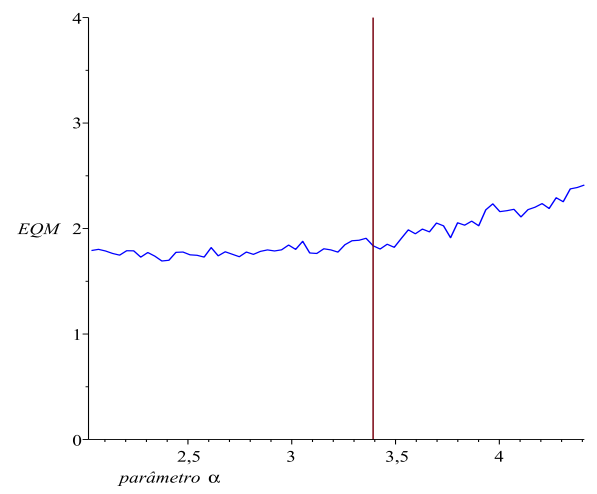

Figura 3: $(0.8654,0.1899, \alpha, 1.2842)$

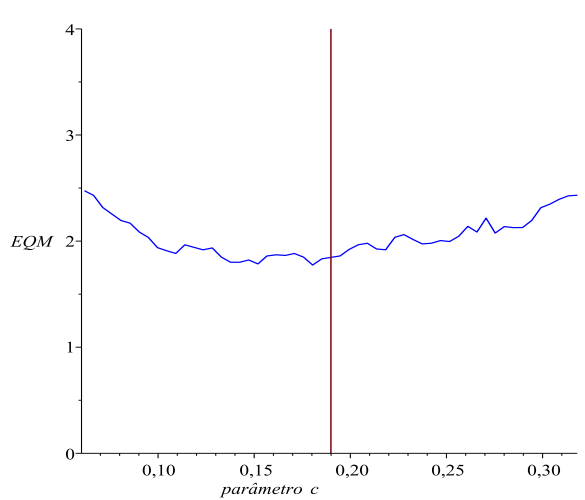

Figura 2: $(0.8654, c, 3.3920,1.2842)$

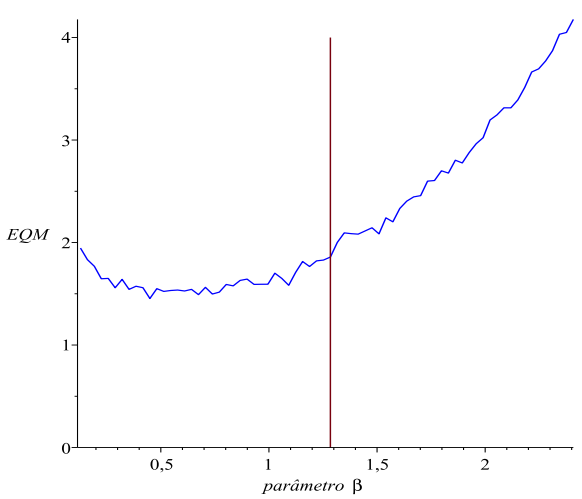

Figura 4: $(0.8654,0.1899,3.3920, \beta)$

\section{Aplicação}

Nesta seção é feita uma aplicação do modelo na análise de dados de precipitações pluviométricas ocorridas na cidade de Passo Fundo, Estado do Rio Grande do Sul.

Os dados explorados correspondem a medições diárias de precipitação pluviométrica (mm) no período de Julho de 2009 a Julho de 2011, totalizando 730 observações. Os dados foram coletados pelo Laboratório de Meteorologia Aplicada à Agricultura da Embrapa Trigo, Passo Fundo - RS, (latitude: $28^{\circ} 15^{\prime} 46^{\prime \prime}$ S; longitude: $52^{\circ} 24^{\prime} 24^{\prime \prime} \mathrm{W}$; altitude: $684 \mathrm{~m}$ ) e encontram-se disponíveis livremente para download no endereço eletrônico http://www.cnpt.embrapa.br/pesquisa/agromet.

Utilizando as medições do índice pluviométrico, obtém-se os dados sobre período de dias com ocorrência de precipitações $(X)$ e período contíguo de dias sem ocorrência de precipitação $(Y)$. O objetivo é modelar as variáveis $X, Y$ e o período climático $U$. O período climático indica um ciclo climático, formado pela soma dos dias sem chuva com os dias contíguos com chuva, essa quantidade também indica o retorno do período de chuva. 
O ajuste das distribuições foi feito via Método da Máxima Verossimilhança. Se $\left(x_{1}, y_{1}\right), \ldots,\left(x_{n}, y_{n}\right)$ é uma amostra aleatória de $(2.1)$, as estimativas dos parâmetros do modelo são: $\hat{a}=0.8654, \hat{c}=0.1899$, $\hat{\alpha}=3.3920$ e $\hat{\beta}=1.2842$.

As fdps ajustadas de $X, Y$ e $U$ e os respectivos gráficos de probabilidades observadas versus probabilidades esperadas, são apresentadas nas Figuras de 5 a 10 .

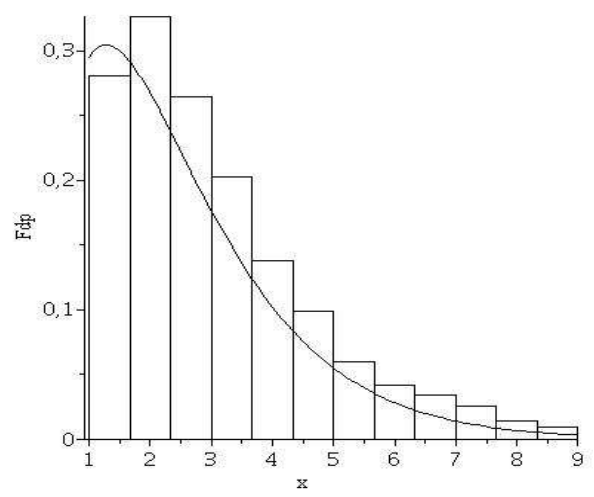

Figura 5: fdp ajustada de $X$

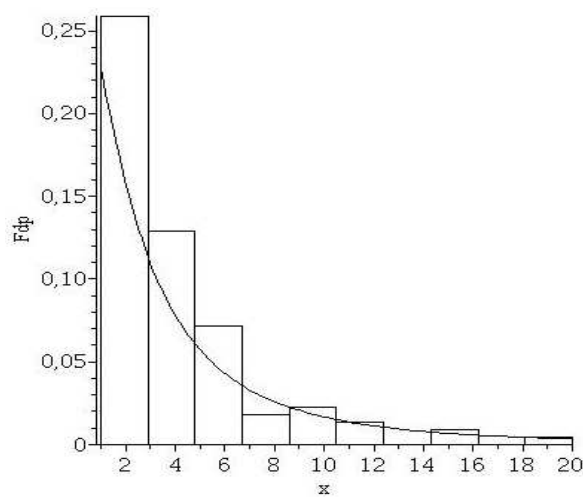

Figura 7: fdp ajustada de $Y$

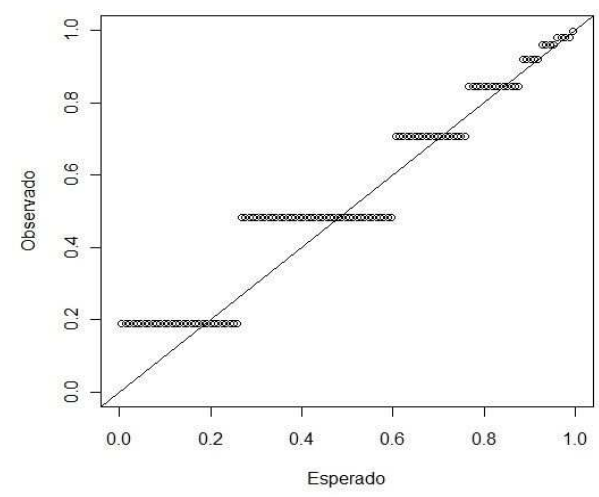

Figura 6: p-pplot para a variável $X$

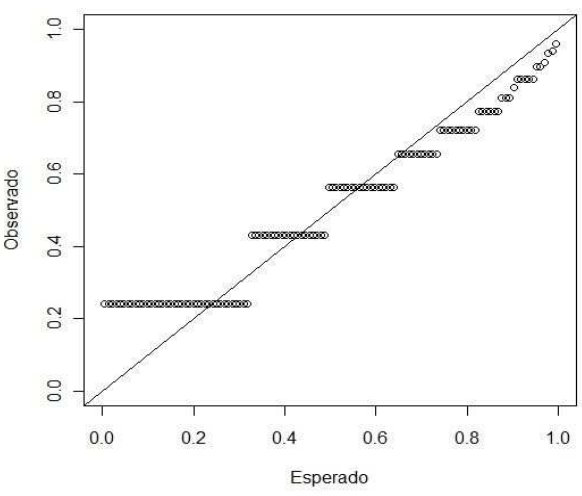

Figura 8: p-pplot para a variável $Y$

Os gráficos das distribuições ajustadas e de probabilidade, Figuras 5 a 10, sugerem um bom ajuste para as variáveis período de chuva $(X)$, período sem ocorrência de chuva $(Y)$ e período climático $(U)$, pelo gama beta tipo II. Deste modo temos que o modelo bivariado gama beta II apresenta-se como uma alternativa na análise de dados de precipitação pluviométrica. 


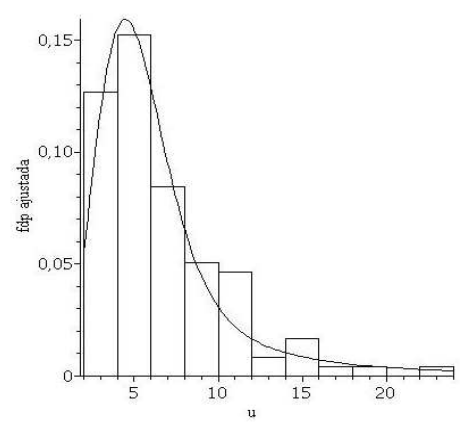

Figura 9: fdp ajustada de $Y$

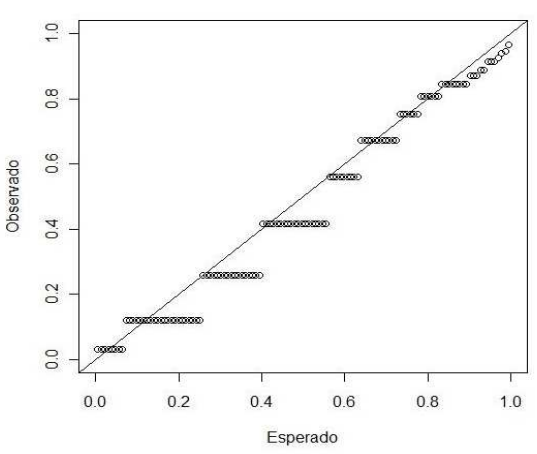

Figura 10: p-pplot para a variável $Y$

\title{
4. Conclusões
}

Considerando que $X$ e $Y$ seguem o modelo bivariado gama beta II, foi possível deduzir as distribuições exatas e os momentos das variáveis aleatórias $U=X+Y$, $P=X Y$ e $Q=X /(X+Y)$ utilizando funções especiais. A estimação dos parâmetros do modelo pelo Método da Máxima Verossimilhança e suas propriedades, podem computadas utilizando métodos numéricos.

A aplicação do modelo na análise de dados de precipitações ocorridas na cidade de Passo Fundo do Estado do Rio Grande do Sul apresentou resultados satisfatórios, levando-se em consideração que os critérios gráficos de qualidade de ajuste indicaram uma boa adequação do modelo aos dados observados.

\begin{abstract}
Bivariate gamma distributions have been used successfully on modeling hydrological processes. In this work, supposing that $X$ and $Y$ follow the gamma beta II bivariate gamma model, we deduce the exact distributions of the functions $U=X+Y, P=X Y$ and $Q=X /(X+Y)$, as well as their respective moments. Those functions describe important hidrological variables. An application of the results is provided to rainfall data from Passo Fundo - RS.
\end{abstract}

Keywords. Gamma beta II distribution, combination of random variables, rainfall.

\section{Referências}

[1] T. Izawa, Two or multi-dimensional gamma-type distribution and its application to rainnfall data, Meteorological Research Institute, 15 (1965), 167-200.

[2] N. Balakrifhnan, C.D. Lai, "Continuous bivariate distributions", Springer Vergland, 2009.

[3] R. Beals, R. Wong, "Special functions", Cambridge University, New York, 2010. 
[4] A.K. Gupta, S. Nadarajah, Sums, products and ratios for Mckay's bivariate gamma distribution, Mathematical and Computer Modelling, 43 (2006), 185193.

[5] M.V. Jambunathan, Some properties of beta and gamma distributions, The Annals of Math. Stat., 25 (1954), 401-405.

[6] S. Nadarajah, Products and ratios for a bivariate gamma distribution, Journal of the American Statistical Association Applied Mathematics and Computing, 24, No. 1 (2005), 581-595.

[7] S. Nadarajah, Bivariate gamma exponential distribution with application to drought data, Journal Applied Mathematical and Computer Modelling, 24, No. 1 (2007), 221-130.

[8] S. Nadarajah, Bivariate pareto model for drought, Stochastic Environmental Research and Risk Assessment, 23, No. 6 (2008), 811-822.

[9] S. Nadarajah, A.K. Gupta, Cherian's bivariate gamma distributioins as a model for drought data, Agrociencia, 40, No. 4 (2006), 483-490.

[10] S. Nadarajah, A.K. Gupta, Friday and Patil's bivariate exponential distributioin with application to drought data, Water Resource Management, 20, No. 5 (2006), 749-759.

[11] S. Nadarajah, S. Kotz, Sums, products and ratios for downton's bivariate exponential distribution, Stochastic Environmental Research and Risk Assessment, 20, No. 3 (2006), 164-170.

[12] K. Oldham, J. Myland, J. Spanier, "An atlas of functions: with equator, the atlas function calculator", Springer Vergland, Canadá, 2009.

[13] A.P. Prudnikov, O.I. Marichev, "Integrals and series", Gordon and Breach, Amsterdam, 1998.

[14] A.P.C.M. Silva, J.A. Rodrigues, L.M. Chaves, D.J. Souza, Sums, Products and Ratios for Crovelli's Bivariate Gamma Distribution, Water Resources Management, 27, No. 5 (2013), 1363-1376.

[15] S. Yue, A bivariate gamma distribution for use in multivariate flood frequency analysis, Journal of Hydrology, 15, No. 6 (2001), 1033-1045.

[16] S. Yue, T.B.M.J. Ouarda, B. Bobee, A review of bivariate gamma distributions for hydrological application, Journal of Hydrology, 246, No.1 (2001), 1-18. 